VII.

Aus der med. Klinik (Vorstand: Prof. Dr. A. Gluziński) und dem Institut für med. Chemie (Vorstand: Prof. Dr. St. Bądzyński) der Universität in Lemberg.

\title{
Ueber den Einfluss der Atophandarreichung auf die Urochromausscheidung.
}

Von

Dr. Witold Skórezewski.

Die Störungen der Oxydation des Schwefels im Organismus nach Darreichung von Atophan, welche ich mit Sohn (2) stets bei unseren Stoffwechselversuchen beobachtete, zwangen mich zur Ausführung solcher Versuche, welche die Erage erklären-könnten: durch welche schwefelhaltige organische Verbindung eine Vermehrung des neutralen Schwefels im Atophanharne verursacht werde.

Die Versuche von Gawinski wiesen auf die Oxyproteinsäuren, als auf solche Körper, welche den gesammten neutralen Schwefel des Harnes enthalten, hin. Meine Bestimmungen des Stickstoffs und des Schwefels des Oxyproteinsäuren-Bariumsyrups (3) vor und nach der Atophandarreichung weisen Störungen in den Atophanperioden auf. Wenn jedoch die Stickstoffmenge nur eine geringe Vergrösserung erfährt, erhalten wir in dem Schwefelgehalte des Bariumsyrups eine viel eminentere Zunahme. Es konnte also aus dieser Verschiebung des Verhältnisses der Schwefelmenge zum Stickstoff im Bariumsyrup der Oxyproteinsäuren auf Kosten der letzteren auf die Vermehrung der schwefelreicheren Glieder der 0xyproteinsäurengruppe geschlossen werden. Schon damals habe ich auf die Wahrscheinlichkeit der Vermehrung des Urochroms hingewiesen, in der jetzigen Publication stellte ich Versuche bei vier Kranken an, die in den lolgenden Tabellen ausführlich notirt sind.

Die Bestimmungen des Urochroms wurden in den täglichen Mengen frischen Urins nach der Methode von Browinski und Dabrowski (1) (Bulletin de l'académie de sciences de Cracovie XLVIII. 1908) ausgeführt. Die Methode beruht auf der Fällung des Urochroms durch Kupferacetat, auf der Zersetzung des Niederschlages mit Schwefelwasserstoff und auf der Bestimmung der Menge des Urochroms in der wässerigen Lösung nach Entfernung des überschüssigen Schwefelwasserstoffes unter Verwerthung seiner reducirenden Eigenschaften. Urochrom reducirt nämlich Jodsäure und das dabei frei werdende Jod wird mit Kohlenschwefelstoff extrahirt und sein Gehalt mit $\frac{\mathrm{n}}{100}$ Thiosulphatlösung bestimmt. 
Stickstoff wurde nach Kjeldahl, Harnsäure nach Wörner's Methode bestimnt, Mineralschwefel wurde mit Chlorbarium gefällt. Die Veraschung bei der Bestimmung des neutralen Schwefels mit Natrium peroxydatum nach der Methode von Modrakowski ausgeführt.

Versuch I. Protokoll No. 5. Bett 8. J. H., Krankenpflegerin, 45 Jahre alt, aufgenommen am 16. 11. Diagnose: Arthritis urica. Condensatio apicis sinistri. Vom 18. 11. purinfreie Diät. Vom 1.12. bis 20.12. verweilt sio 2 Stunden täglich im Emanatorium. (Tabelle I.)

Den 11. 12. bekam sie $3 \mathrm{~g}$ Atophan in 3 Dosen. Tabelle I zeigt deutlich die mit dem neutralen Schwefel parallel gehende Vermehrung des Urochroms nach Verabreichung von Atophan.

Tabelle I.

\begin{tabular}{|c|c|c|c|c|c|c|c|c|c|c|}
\hline Datum & $\begin{array}{l}\text { Harn- } \\
\text { meage }\end{array}$ & $\begin{array}{c}\mathrm{Cl} \\
\text { als } \\
\mathrm{NaCl}\end{array}$ & 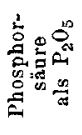 & $\begin{array}{l}\text { Stick- } \\
\text { stoff }\end{array}$ & $\begin{array}{l}\text { Harn- } \\
\text { säure }\end{array}$ & $\begin{array}{l}\text { Uro- } \\
\text { chrom }\end{array}$ & $\begin{array}{c}\text { Mineral- } \\
\text { schwefel } \\
\text { als } \mathrm{SO}_{3}\end{array}$ & $\begin{array}{c}\text { Neutral- } \\
\text { sehwefel } \\
\text { als } \mathrm{SO}_{3}\end{array}$ & $\begin{array}{c}\text { VorhäItniss } \\
\text { des neutr. } S . \\
\text { zurn Ge- } \\
\text { sammt-S. in } \\
\text { pCt. }\end{array}$ & $\begin{array}{c}\text { Be- } \\
\text { merkungen }\end{array}$ \\
\hline 8. 12. & 1340 & 5,75 & 3,95 & 8,61 & 0,1899 & 0,1474 & 0,5963 & 0,0434 & 6,7 & \multirow{6}{*}{$3 \mathrm{~g} \mathrm{Atophar}$} \\
\hline 9.12. & 1000 & 6,2 & 1,7 & 4,48 & 0,218 & 0,2045 & 0,891 & 0,0745 & 7,7 & \\
\hline 10.12. & 1100 & 5,39 & 2,14 & 4,71 & 0,1782 & 0,1131 & 0,8206 & 0,1463 & 15,1 & \\
\hline 11.12. & 1940 & 6,6 & 1,98 & 7,2 & 0,5745 & 0,2428 & 1,1601 & 0,2619 & 18,4 & \\
\hline 12.12 . & 1300 & 6,63 & 1,77 & 6,24 & 0,2834 & 0,2614 & 1,0517 & 0,3107 & 22,8 & \\
\hline 13.12 . & 1200 & 5,4 & 1,01 & 3,42 & 0,1104 & 0,2242 & 1,1299 & 0,0876 & 7,1 & \\
\hline
\end{tabular}

Versuch II. Protokoll No. 3. Bett 10. R. K., Maurer, 44 Jahre alt, aufgenommen am 6. 10 . Diagnose: Rheumatismus chronicus articulationis humeri sin. Neuritis plexus brachialis sin. in individuo cum induratione apicis sinistri et concretionibus pleuralibus sin. intercurrente otitide media sin. verisimiliter tuberculosa. (Tabelle II.)

Tabelle II.

\begin{tabular}{|c|c|c|c|c|c|}
\hline Datum & Harnmenge & Stickstoff & Harnsäure & Urocbrom & Bemerkungen \\
\hline $\begin{array}{l}14.11 . \\
15.11 . \\
16.11 .\end{array}$ & $\begin{array}{r}880 \\
1110 \\
1260\end{array}$ & $\begin{array}{l}4,99 \\
9,79 \\
8,47\end{array}$ & $\begin{array}{c}-\overline{4068} \\
0,4377\end{array}$ & $\begin{array}{l}0,1542 \\
0,2027\end{array}$ & \\
\hline Mittel & & & & 0,1785 & \\
\hline $\begin{array}{l}17.11 . \\
18.11 . \\
19.11 .\end{array}$ & $\begin{array}{r}1120 \\
880 \\
1220\end{array}$ & $\begin{array}{r}8,81 \\
7,88 \\
12,21\end{array}$ & $\begin{array}{l}0,4863 \\
0,4137 \\
0,5499\end{array}$ & $\begin{array}{l}0,3435 \\
0,2367 \\
0,2116\end{array}$ & $\begin{array}{c}3 \mathrm{~g} \text { Atophan. } \\
\text { do. } \\
\text { do. }\end{array}$ \\
\hline Mittel & & & & 0,2639 & \\
\hline
\end{tabular}

Versuch III. Protokoll No. 114. Bett 7. J. M., Schuldiener, 52 Jahre alt, aufgenommen am 22. 11. Diagnose: Neurasthenia in individuo luetico. Gemischte Diät vom 5. 12. bis 7. 12. je $3 \mathrm{~g}$ Atophan täglich. (Tabelle III.)

Versuch IV. Protokoll No. 115. Bett 5. S. C., Student, 23 Jahro alt, aufgenommen am 22. 11. Diagnose: Pleuropericarditis sicca in individuo cum induratione apicum utriusque. Gemischte Diät vom 16. 12. bis 18. 12. je $3 \mathrm{~g}$ Atophan täglich. Während des Versuches fieberfrei. (T'abelle IV.) 
Ueber den Einfluss der Atophandarreichung auf die Urochromausscheidung. 115

Tabelle III.

\begin{tabular}{|c|c|c|c|c|c|c|c|c|}
\hline Datum & $\begin{array}{l}\text { Harn- } \\
\text { menge }\end{array}$ & $\begin{array}{l}\text { Stick- } \\
\text { stoff }\end{array}$ & $\begin{array}{l}\text { Harn- } \\
\text { säure }\end{array}$ & $\begin{array}{l}\text { Uro- } \\
\text { chrom }\end{array}$ & $\begin{array}{c}\text { Mineral- } \\
\text { schwefel } \\
\text { als } \mathrm{SO}_{3}\end{array}$ & $\begin{array}{c}\text { Neutral- } \\
\text { schwefel } \\
\text { als } \mathrm{SO}_{3}\end{array}$ & $\begin{array}{l}\text { Verhältniss d. } \\
\text { neutr. S. zum } \\
\text { Gesammt-S. } \\
\text { in pCt. }\end{array}$ & $\begin{array}{c}\mathrm{Be}- \\
\text { merkungen }\end{array}$ \\
\hline $\begin{array}{l}\text { 2. } 12 . \\
\text { 3. } 12 . \\
\text { 4. } 12 .\end{array}$ & $\begin{array}{l}1000 \\
1190 \\
1090\end{array}$ & $\begin{array}{r}11,17 \\
10,41 \\
8,30\end{array}$ & $\begin{array}{l}0,3498 \\
0,3297 \\
0,3171\end{array}$ & $\begin{array}{l}0,2483 \\
0,1957 \\
0,2039\end{array}$ & $\begin{array}{l}1,5728 \\
1,7374 \\
1,3233\end{array}$ & $\begin{array}{l}0,1248 \\
0,2644 \\
0,10406\end{array}$ & $\begin{array}{r}7,3 \\
13,2 \\
9,6\end{array}$ & \\
\hline Mittel & & & & 0,2159 & & & & \\
\hline $\begin{array}{l}\text { 5. } 12 . \\
6.12 . \\
7.12 .\end{array}$ & $\begin{array}{r}980 \\
1063 \\
1140\end{array}$ & $\begin{array}{l}8,19 \\
9,09 \\
8,06\end{array}$ & $\begin{array}{l}0,6438 \\
0,4611 \\
0,5013\end{array}$ & $\begin{array}{l}0,2925 \\
0,2948 \\
0,2137\end{array}$ & $\begin{array}{l}1,2427 \\
1,1140 \\
1,1168\end{array}$ & $\begin{array}{l}0,2911 \\
0,1821 \\
0,1790\end{array}$ & $\begin{array}{l}18,8 \\
14,5 \\
13,0\end{array}$ & $\begin{array}{c}3 \mathrm{~g} \text { Atophan. } \\
\text { do. } \\
\text { do. }\end{array}$ \\
\hline Mittel & & & & 0,267 & & & & \\
\hline
\end{tabular}

Tabelle IV.

\begin{tabular}{|c|c|c|c|c|c|c|c|c|}
\hline Datum & $\begin{array}{l}\text { Harn- } \\
\text { menge }\end{array}$ & $\begin{array}{l}\text { Stick- } \\
\text { stoff }\end{array}$ & $\begin{array}{c}\text { Harn- } \\
\text { säure }\end{array}$ & $\begin{array}{l}\text { Uro- } \\
\text { chrom }\end{array}$ & $\begin{array}{c}\text { Mineral- } \\
\text { schwefel } \\
\text { als } \mathrm{SO}_{3}\end{array}$ & $\begin{array}{c}\text { Neutral- } \\
\text { schwefel } \\
\text { als } \mathrm{SO}_{3}\end{array} \mid$ & $\begin{array}{c}\text { Verhältniss d. } \\
\text { neutr. S. zum } \\
\text { Gesammt-S. } \\
\text { in pCt. }\end{array}$ & $\begin{array}{c}\mathrm{Be}- \\
\text { merkungen }\end{array}$ \\
\hline $\begin{array}{l}13.12 . \\
14.12 . \\
15.12 .\end{array}$ & $\begin{array}{l}1586 \\
1820 \\
2100\end{array}$ & $\begin{array}{l}15,73 \\
16,61 \\
13,46\end{array}$ & $\begin{array}{l}0,6594 \\
0,6825 \\
0,6486\end{array}$ & $\begin{array}{l}0,2896 \\
0,3808 \\
0,5739\end{array}$ & $\begin{array}{l}2,6407 \\
2,5462 \\
2,7371\end{array}$ & $\begin{array}{l}0,3416 \\
0,3946 \\
0,3864\end{array}$ & $\begin{array}{l}11,3 \\
13,4 \\
12,3\end{array}$ & \\
\hline Mittel & & & & 0,4148 & & & & \\
\hline $\begin{array}{l}16.12 . \\
17.12 . \\
18.12 .\end{array}$ & $\begin{array}{l}1800 \\
1215 \\
1820\end{array}$ & $\begin{array}{l}16,37 \\
15,0 \\
12,91\end{array}$ & $\begin{array}{l}1,35 \\
0,8676 \\
0,7947\end{array}$ & $\begin{array}{l}0,4623 \\
0,5526 \\
0,7288\end{array}$ & $\begin{array}{l}2,1438 \\
1,7314 \\
1,745\end{array}$ & $\begin{array}{l}0,2815 \\
0.4629 \\
0,6024\end{array}$ & $\begin{array}{l}11,6 \\
21,0 \\
25,6\end{array}$ & $\begin{array}{c}3 \mathrm{~g} \text { Atophan } \\
\text { do. } \\
\text { do. }\end{array}$ \\
\hline Mittel & & & & 0,5812 & & & & \\
\hline
\end{tabular}

Die Ergebnisse unserer Versuche zusammenstellend gelangen wir zu dem Schlusse, dass das Einnehmen von Atophan eine stärkere Urochromausscheidung nach sich zieht. Alle diese Versuche weisen eine parallele Vermehrung des neutralen Schwefels und des Urochroms nach Atophandarreichung auf.

Die hier bemerkten Thatsachen können ein wenig Licht in das bis jetzt wenig bekannte Gebiet der Oxydationsstörung bringen, da die bis jetzt beobachtete Vermehrung der Oxyproteinsäuren bei Fieberkrankheiten, sowie nach reichlicherem Fleischgenuss stets eine gleichartige Vermehrung des Stickstoffs und des Schwefels des Oxyproteinsäurensyrups nach sich zieht.

\section{Literaturbericht.}

1) Józef Browiński i Stefan Dąbrowski, O metodzic ilościowego określenia żoltego podstawowego barwika moczu (urochroum). Rozprawy Wydzialu mat. przyrodę. Akad. umicj. w Krakowie. T. XLVIII. B.

2) W. Skórczewski und J. Sohn, Stoffwechselversuche bei Atophandarreichung. Diese Zeitschr. Bd. 11.

3) W. Skórczewski, Warum vergrössert Atophan die Ausscheidung dèr Harnsäure? Diese Zeitschr. Bd. 11. 